\title{
A NOTE ON RINGS WITH CENTRAL NILPOTENT ELEMENTS
}

\section{N. HERSTEIN}

The theorem proved in this note, and its corollary, are designed to improve, and in a sense to bring to a more satisfactory completion, a theorem which we proved in [1].

We prove the

TheOREM. Let $R$ be a ring such that for every element $x$ in $R$ there exists an integer $n=n(x)$, and a polynomial $p(t)=p_{x}(t)$ with integer coefficients which depend on $x$, such that $x^{n+1} p(x)=x^{n}$. If further all the nilpotent elements of $R$ are in the center of $R$, then $R$ is commutative.

Proof. Since $x^{n+1} p(x)=x^{n}$, we have that $\left(x^{2} p(x)-x\right) x^{n-1}=0$ (we can assume that $n>1$ for this could always be achieved by multiplying both sides of the equation by $x)$. Now, each term of $\left(x^{2} p(x)-x\right)^{n-1}$ involves $x$ to a power which is at least $n-1$; therefore $\left(x^{2} p(x)-x\right)^{n}$ $=\left(x^{2} p(x)-x\right)\left(x^{2} p(x)-x\right)^{n-1}=0$. Since $x^{2} p(x)-x$ is nilpotent, by assumption it must lie in the center of $R$. This is true for every $x$ in $R$, so it follows from [2] that $R$ is commutative.

COROLlaRY. Let $R$ be a ring such that every element of $R$ generates a finite subring. If the nilpotent elements of $R$ are all in the center, then $R$ is commutative.

Proof. Since $x$ in $R$ generates a finite subring, $x^{n}=x^{m}$ for some $n>m$, so the corollary is immediate from the theorem. This corollary is a direct generalization of the second theorem in [1].

\section{REFERENCES}

1. I. N. Herstein, A proof of a conjecture of Vandiver, Proc. Amer. Math. Soc. vol. 1 (1950) pp. 370-371.

2. - - The structure of a certain class of rings, Amer. J. Math. vol. 75 (1953) pp. $864-871$.

University of Pennsylvania

Reccived by the editors December 15, 1953. 